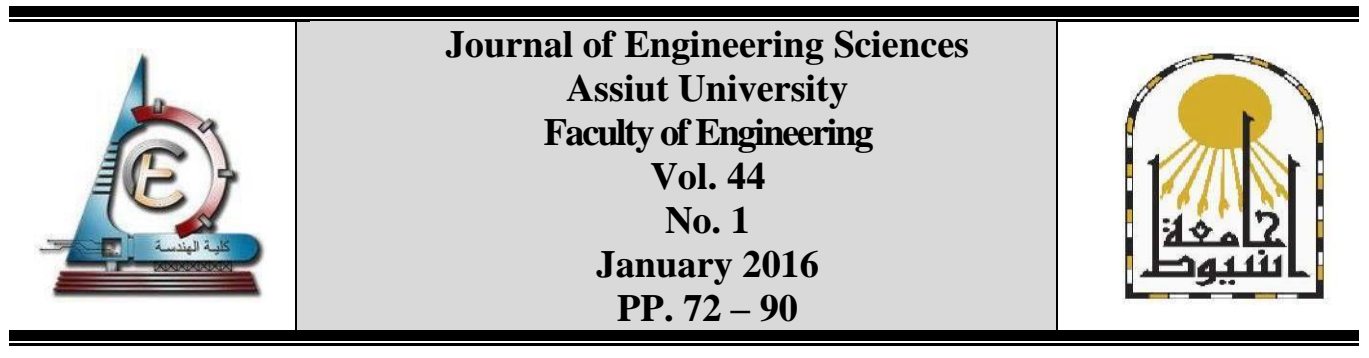

\title{
EFFECT OF MISALIGNMENT ON THE DURABILITY OF GAS-LUBRICATED FOIL THRUST BEARING
}

\author{
Abdelrasoul M. Gad \\ Department of Mechanical Engineering, Faculty of Engineering, Assiut University, Egypt. \\ E-mail: ammgad76@aun.edu.eg
}

Received 17 November 2015; Accepted 20 December 2015

\begin{abstract}
The present study aims to evaluate the effects of static and dynamic angular misalignments of the runner surface on the performance of a gas-lubricated foil thrust bearing. The responses of the bearing load capacity, viscous power loss, and the stiffness and damping coefficients of the gas film to small angular misalignments are thoroughly analyzed. The compressible Reynolds equation along with the Couette Approximation technique is used to model the flow in the gas film. The deformation of the compliant bearing is calculated with a robust analytical model, and small perturbations method is used to calculate the dynamic coefficients of the gas film. The results show that misaligned foil thrust bearings are capable of developing a restoring moment sufficient enough to withstand the imposed mechanical distortions. Furthermore, the enhanced hydrodynamic effect ensures a stable operation of the misaligned foil thrust bearing at very thin gas films.
\end{abstract}

Keywords: Angular misalignment, foil thrust bearing, thermal elastohydrodynamic lubrication, static and dynamic characteristics

\section{Introduction}

Foil gas bearings provide prominent merits over the conventional rolling element and oil bearings such as the endurance of high temperature, endurance of foreign matter, improved damping, and the ability to accommodate assembly misalignments [1, 2]. Therefore, radial foil air bearings have been applied successfully to aircraft Air Cycle Machines (ACMs), auxiliary power units (APU) its applicability in cryogenic turboexpanders, turbo-alternators, turbochargers, and automotive gas turbine engines have been demonstrated [3,4]. On the other hand, the ability of gas foil thrust bearing to support the axial loads generated due to the pressure difference between compressor and turbine sides in high speed oil-free turbomachinery was assured [5, 6]. Figure 1 shows the schematic representation of a typical single-acting foil thrust bearing assembly is shown in Figure 1. The resilient bearing is composed of a smooth top foil resting on a number of flexible and independent bump foil strips with different number of bumps. 


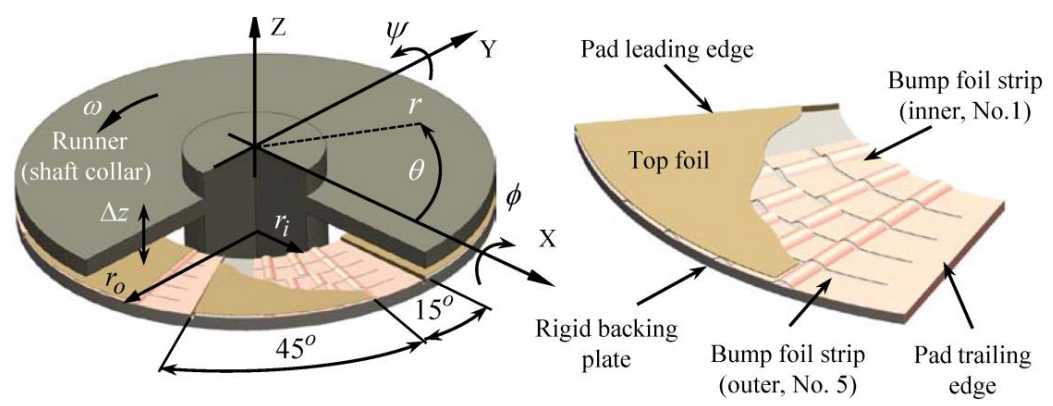

(a) Coordinate system and geometry of foil thrust bearing

(b) Single bearing pad with the top foil partially removed



(c) Gas film geometry of foil thrust bearing



(d) Statically-misaligned foil thrust bearing

Fig. 1. Schematics of the geometry of misaligned foil thrust bearing

Several important studies were performed on gas foil thrust bearings. An early study by Heshmat et al. [1, 7] has investigated the effects of geometrical, structural, and operational parameters on the performance of foil thrust bearing of simple geometry. Later, Iordanoff [8] introduced a method to rapidly design the foil thrust bearing for maximum load carrying capacity. A comprehensive analysis of foil thrust bearing was conducted by Bruckner [9] to investigate the thermal effects on the static characteristics of foil thrust bearing.

Recently, Lee and $\operatorname{Kim}[10,11]$ have thoroughly analyzed the thermal elastohydrodynamic performance of a foil thrust bearing with a newly-designed compliant bearing structure. Gad and Kaneko [12] have introduced a new analytical model to calculate the deformations of bump-type compliant bearings which was applied successfully to predict the static performance characteristics of "second generation" foil thrust bearings. Most recently, Gad and Kaneko [13-15] have studied the static and dynamic characteristics of foil thrust bearing considering the centrifugal forces with new architectures of the compliant bearing of "second generation" foil structure.

Further, successful experimental works have been conducted on "second generation" foil thrust bearing by Dykas [16], Dickman [17], and Bruckner [18] to investigate the static characteristics of the bearing at a wide range of operating conditions.

When used to support axial loads between compressor and turbine sides in turbomachinery applications, foil thrust bearing is located between two radial foil bearings. In practical situations, it extremely difficult to ensure a zero static misalignment between the two radial bearings and hence, the runner surface of foil thrust bearing cannot be 
maintained parallel to the bearing surface; in fact, the runner (shaft collar) exhibits a static angular misalignment in one or more directions, see Figure 1(d). Furthermore, even if the static misalignment is reduced to a minimum, the non-symmetric thermal distortions of the two radial foil bearings can result in a significant rotor misalignment.

However, almost all previous analytical and experimental approaches have neglected the effect of static angular misalignment of the runner on the performance of foil thrust bearings. Very few studies have handled the problem of misaligned foil thrust bearings. Among these studies is the work of Park et al. [19] in which the performance characteristics of foil thrust bearing with "first generation" bump foil structure were evaluated. The bearing load carrying capacity and the viscous friction torque of a statically-misaligned runner were analyzed. Also, Lee and Kim [10] have included the effect of runner-bearing misalignment on the performance of hybrid foil thrust bearing.

Actually, any small angular misalignment of the bearing runner greatly affects the gas film geometry of foil thrust bearings and, consequently, affects its static and dynamic performance characteristics.

Therefore, the objective of the present study is to evaluate the effect of both static and dynamic angular misalignments of the bearing runner on the static and dynamic performance characteristics of foil thrust bearing. The studied foil thrust bearing has a compliant structure of the "second generation" type that was experimentally investigated by Dykas [16], Dickman [17], and Bruckner [18] and was proven to have superior performance features over other architectures; however, the previous studies on this type of bearing have neglected the possibility of the bearing runner static misalignments. The top foil of each bearing pad is clamped at the pad leading edge while the bump foil strips are clamped at the pad trailing edge; see Figure 1(b). Moreover, as the thermal features greatly affect the performance characteristics of foil bearing, a simplified energy equation is used to calculate the temperature distribution in the gas film. The details of the mathematical model are described in the following sections.

\section{Theoretical model}

Similar to all fluid-structure interaction problems, foil bearing simulations require the coupling between the gas film pressure and the compliant structure deformations. The hydrodynamic pressure on the gas film causes the bearing surface to deform which in turn acts to modulate the gas film pressure; this interaction continues until a steady state condition is prevailed.

\subsection{Model of the gas film}

In the present simulations, the two-dimensional compressible Reynolds equation is used to model the flow in the gas film between the rotating shaft collar and the smooth top foil. The gas is assumed to be ideal and the equation of state is used to express the gas density in Reynolds equation in terms of the gas pressure and temperature. Then, the flowing dimensionless quantities are used to convert the Reynolds equation into a non-dimensional form:

$$
\bar{p}=\frac{p}{p_{a}}, \bar{r}=\frac{r}{r_{o}}, \bar{h}=\frac{h}{h_{o}}, \bar{\mu}=\frac{\mu}{\mu_{a}}, \bar{t}=t v, \gamma=\frac{v}{\omega}
$$


and, the Reynolds equation in non-dimensional form, after dropping the bar sign is expressed as:

$$
\frac{\partial}{\partial r}\left(\frac{r p h^{3}}{T \mu(T)} \frac{\partial p}{\partial r}\right)+\frac{1}{r} \frac{\partial}{\partial \theta}\left(\frac{p h^{3}}{T \mu(T)} \frac{\partial p}{\partial \theta}\right)=\Lambda \frac{\partial}{\partial \theta}\left(\frac{r p h}{T}\right)+2 \Lambda \gamma \frac{\partial}{\partial t}\left(\frac{r p h}{T}\right)
$$

where $\Lambda$ is the bearing number defined as:

$$
\Lambda=\frac{6 \mu_{a} \omega}{p_{a}}\left(\frac{r_{o}}{h_{o}}\right)^{2}
$$

As the shaft collar is tilted around the $\mathrm{X}$ and $\mathrm{Y}$ axes, the thickness of the gas film changes in both radial and circumferential directions over each bearing pad. Hence, from Figure 1, the dimensionless film thickness $(h)$, can be expressed as;

$$
h(r, \theta)= \begin{cases}1+H\left(1-\theta / \theta_{c}\right)-\phi(r \sin \theta)+\psi(r \cos \theta)+\bar{\delta}(r, \theta), & 0 \leq \theta \leq \theta_{c} \\ 1-\phi(r \sin \theta)+\psi(r \cos \theta)+\bar{\delta}(r, \theta), & \theta_{c}<\theta \leq \theta_{p}\end{cases}
$$

where, $\phi$ and $\psi$ are the static angular misalignments around $\mathrm{X}$ and $\mathrm{Y}$ axes, respectively. Also, $\bar{\delta}(r, \theta)=\delta / h_{o}$ and $H=h_{1} / h_{o}$ are the dimensionless structure deflection and film thickness ratio, respectively.

In order to obtain the gas film pressure, Equation (1) is integrated over each bearing pad assuming ambient pressure at all boundaries.

\subsection{Model of the gas film temperature}

The Couette Approximation technique that was developed by Pinkus and Bupara [20] is used to calculate the temperature distribution in the gas film. The technique allows the decoupling between the energy equation and Reynolds equation and hence, each equation can be solved independently. The underlying basic assumption is that the effect of pressure gradients on the shear rate of the fluid is much smaller compared with the runner drag effect. It is important to point out that the heat generated in the gas film is assumed to be completely carried away by the gas flow. Thus, the temperature distribution in the air film can be expressed as, [20];

$$
T(r, \theta)=\beta+\left(T_{i n}-\beta\right) e^{G(r, \theta)}
$$

where $G(r, \theta)$ is a non-dimensional function expressed as;

$$
G(r, \theta)=\left(\frac{2 \alpha \omega}{\rho C_{p}}\right)\left(\frac{r}{c}\right)^{2} \int_{0}^{\theta}\left(\frac{c}{h}\right)^{2} d \theta
$$


The constants $T_{i n}$ and $C_{p}$ represent the gas entrance temperature and its specific heat, respectively. At the temperature operating range of foil thrust bearing, the gas viscosity can be assumed to vary linearly with temperature as follows:

$$
\mu(T)=\alpha(T-\beta)
$$

where, $\alpha$ and $\beta$ are the coefficients of the linear viscosity-temperature relationship and their values can be obtained through a linear curve fitting of the given viscositytemperature data $[11,21]$. Hence, Equation (3) is solved for the gas film temperature over each bearing pad assuming a constant ambient temperature at all boundaries.

\subsection{Model of the bump foil}

Modeling foil gas bearings is a troublesome problem mainly because of the difficulties encountered when calculating the deformation of the compliant bearing under the developed hydrodynamic pressure. Several important approaches were introduced to model the structural stiffness of bump-type foil bearings. Among these models, the oneequation model developed by Walowit and Anno [22], the widely used two-equation model developed by Iordanoff [8], the analytical and more elaborate model of $\mathrm{Ku}$ and Heshmat [23], the inter-connected springs model of Le Lez, et al. [24], the link-spring model of Feng and Kaneko [25], the interesting bump-foil analysis of Hryniewicz et al. [26], the bump-root bending approaches of Kim et al. [27], and the most recent model introduced by the authors, Gad and Kaneko [12] which is adopted in the present study.



Intermediate bump model

End bump model

Fig. 2. Force analysis of individual bumps in bump foil strip [12]

As shown in Figure 2, the bump foil model assumes that the displacement of bumps in each bump-foil strip is from the fixed end towards its free end, and the top foil is assumed to be a thin deformable membrane that follows the bump foil deflection. Moreover, the model assumes that the bump ends do not separate from the rigid backing plate, however the flat segment between bumps may deflect laterally. In the schematic representation of Figure 2, the models of an intermediate bump ( $i$ ) and the end bump ( $N)$ are shown: here, $(\mu)$ represents the coefficient of friction between the bump-foil strip and the rigid backing plate; also, $(\eta)$ represents the friction coefficient between the bump-foil strip and the top foil; the force $(W)$ represents the concentrated load per unit lateral width of the strip. The forces $\mathrm{Fr}$ and $\mathrm{Fl}$ represent the normal reaction forces at the right and left ends of the bump, respectively. Also, $M r$ and $M l$ represent the bending moments at the right and left ends of the bump, respectively. The force $(\mathrm{Pr})$ is the compressive force transmitted through the flat segments between bumps. The Castigliano's second theorem is used 
sequentially to calculate the structural deformations of the bearing; see Ref. [12] for the detailed analytical model.

For Generation II foil thrust bearings, the staggered pattern of bumps distribution is expected to decrease the effect of top foil sagging between bumps. Therefore, top foil sagging between successive bumps is assumed to be negligible in the present study. Further, the deflection of the top foil between any two successive bumps in the same bump-foil strip is assumed to vary linearly across the distance between bumps.

\section{Numerical techniques}

In order to calculate the gas film pressure, the non-dimensional form of Reynolds equation is discretized using the Finite Difference Method and the Newton-Raphson method is used to linearize the discretized equations. The linearized equations are then solved iteratively for the dimensionless pressure distribution in the gas film using TriDiagonal Matrix Algorithm (TDMA) along with the Alternating Direction Implicit (ADI) method to accelerate the solution convergence. The obtained pressure is then used to estimate the concentrated load over each bump and hence, the deflection of each bump is calculated using the bump foil model described in Section 2.3. In order to obtain the top foil deflection at all intermediate grid points between bumps vertexes, a smooth bilinear interpolation method is used and, consequently, the new gas film thickness at all grid points is determined and a calculation cycle $(m)$ is completed. The new gas film thickness is then fed into both the temperature model, to calculate the gas film temperature, and the Reynolds equation to obtain a new steady state gas film pressure which in turn is used to calculate the bumps deflections and, consequently, a new gas film thickness at the calculation cycle $(m+1)$. This scenario is repeated until a steady state gas film pressure and a corresponding steady state gas film thickness are obtained [13, 15].

The obtained steady state conditions are then used to the bearing static characteristics as follows:

The bearing load carrying capacity $(F)$ is calculated as;

$$
F=\iint_{r} p r d r d \theta
$$

The viscous power loss in the gas film $(P w)$ is calculated as;

$$
P w=\omega \cdot \iint_{r}\left[\frac{h}{2} \frac{\partial p}{\partial \theta}+\frac{\mu_{a} \omega r^{2}}{h}\right] r d r d \theta
$$

The restoring moments ( $M X$ and $M Y$ ) of the gas film are calculated as:

$$
M X=\iint_{r} p r^{2} \sin \theta d r d \theta, \quad M Y=-\iint_{r} p r^{2} \cos \theta d r d \theta
$$

The Simpson's rule is used to evaluate the integrations (6 and 7) numerically. 


\section{Perturbation method}

The small perturbations method is used in this study to calculate the dynamic coefficients of the gas film. Accordingly, the steady state position of the runner is perturbed by small translational displacement $(\Delta z)$, small rotational displacements $(\Delta \phi$ and $\Delta \psi$ ), small translational velocity $(\Delta \dot{z})$, and small rotational velocities $(\Delta \dot{\phi}$ and $\Delta \dot{\psi})$. From Figure 1, the gas film thickness can be expressed as;

$$
h=h_{o}+r[(\psi+\Delta \psi) \cos \theta-(\phi+\Delta \phi) \sin \theta]+\Delta z
$$

and $h_{O}$ stands for the steady state film thickness. The term $(\phi+\Delta \phi)$ represents the static and the dynamic angular misalignments of the runner surface around the $\mathrm{X}$-axis. Similarly, the term $(\psi+\Delta \psi)$ represents the static and the dynamic angular misalignments of the runner surface around the $\mathrm{Y}$-axis.

A set of seven perturbation equations is obtained and expressed in non-dimensional form using suitable parameters [13]. The perturbation equations are discretized using the finite difference method and solved iteratively with tri-diagonal matrix algorithm. Once the pressure perturbations are obtained, the stiffness coefficients of the gas film are calculated as:

$$
\left[\begin{array}{ccc}
K_{z z} & K_{z \phi} & K_{z \psi} \\
K_{\phi z} & K_{\phi \phi} & K_{\phi \psi} \\
K_{\psi z} & K_{\psi \phi} & K_{\psi \psi}
\end{array}\right]=\iint_{r \theta}\left\{\begin{array}{c}
-1 \\
r \sin \theta \\
r \cos \theta
\end{array}\right\}\left\{\begin{array}{lll}
p_{z} & p_{\phi} & p_{\psi}
\end{array}\right\} r d r d \theta
$$

Similarly, the damping coefficients of the gas film are calculated as

$$
\left[\begin{array}{ccc}
C_{z z} & C_{z \phi} & C_{z \psi} \\
C_{\phi z} & C_{\phi \phi} & C_{\phi \psi} \\
C_{\psi z} & C_{\psi \phi} & C_{\psi \psi}
\end{array}\right]=\iint_{r \theta}\left\{\begin{array}{c}
-1 \\
r \sin \theta \\
r \cos \theta
\end{array}\right\}\left\{\begin{array}{lll}
p_{\dot{z}} & p_{\dot{\phi}} & p_{\dot{\psi}}
\end{array}\right\} r d r d \theta
$$

where $p_{u}=\frac{\partial p}{\partial u},(u=z, \phi, \psi, \dot{z}, \dot{\phi}, \dot{\psi})$ are the pressure perturbation components.

\section{Results and discussions}

In the present numerical simulations, the thrust plate of a gas-lubricated foil bearing, shown in Figure 1, is composed of 6 bearing pads. The top foil of each bearing pad is a sector-shaped thin plate clamped at the leading edge of the bearing pad, free at all other edges and extends over a $45^{\circ}$ of the pad angular extension; the remaining $15^{\circ}$ of the pad angular extension is left uncovered as a spacing region between successive bearing pads. Opposite to the top foil clamping, the bump foil is clamped at the pad trailing edge, free at all other edges and extends a $45^{\circ}$ of the over pad angular extension. Hence, only a $30^{\circ}$ sector of the top foil is supported by the bump foil strips, see Figure 1(b). As shown in Figure 1(b), the bump foil is composed of 5 independent bump foil strips with different number of bumps and the end bumps of all bump foil strips are aligned radially with the 
trailing edge of the top foil. For the numerical results presented here, the number of bumps in the bump foil strips are set as 3, 3, 4, 5, and 4 starting from the innermost strip (i.e., strip No.1) towards the outer one (i.e., strip No.5). As a result of the different numbers of bumps in bump foil strips, the angular extension of the converging gas film differs from strip to another; see Figure 1(c). The geometrical parameters of the studied foil thrust bearing and the mechanical properties of the foil material are given in Table 1.

Table 1.

Geometrical parameters of the studied foil thrust bearing.

\begin{tabular}{|l|l|}
\hline Inner radius (mm) & 25 \\
Outer radius (mm) & 50 \\
Number of bearing pads & 6 \\
Bump foil angular extent (degrees) & 45 \\
Top foil angular extent (degrees) & 45 \\
Number of bump foil strips / pad & 5 \\
Top foil thickness, $t_{f}(\mathrm{~mm})$ & 0.1524 \\
Bump foil thickness, $t_{b}(\mathrm{~mm})$ & 0.102 \\
Bump radius, $R(\mathrm{~mm})$ & 1.5875 \\
Bump height, $h_{b}(\mathrm{~mm})$ & 0.508 \\
Young's modulus of bump foil, $E(\mathrm{GPa})$ & 214 \\
Poisson's ratio of bump foil, $v$ & 0.29 \\
\hline
\end{tabular}

For the present predictions, the nominal gas film thickness $\left(h_{o}\right)$ ranges from $0.01 \mathrm{~mm}$ to $0.03 \mathrm{~mm}$ which is the practical operating range for all gas foil bearings [4]. Moreover, the film thickness ratio ( $H$ ) is set as 4 since it yields a maximum load carrying capacity and a lower power loss for foil thrust bearings [12,13]. As Figure 1(a) shows, the shaft collar (i.e., runner) can be tilted around both $\mathrm{X}$ and $\mathrm{Y}$ axes with an angular misalignments of $\Delta \phi$ and $\Delta \psi$, respectively. For simplicity of presentation, the present predictions assume an angular misalignment of the runner around the $\mathrm{X}$-axis only. The maximum tilting angle of the runner attained with a nominal gas film thickness of $0.03 \mathrm{~mm}$ is 480 micro-radians which translate into a minimum gas film thickness as low as 20 percent of the nominal clearance.

\subsection{Static and dynamic characteristics with aligned shaft collar}

As the shaft collar rotates and drags the gas into the converging gap between the top foil and the runner surface, a hydrodynamic pressure is developed in the gas film and the compliant bearing deflects to accommodate the gas pressure; the interaction between the flow pressure and the compliant structure continues until a steady state condition prevails. 


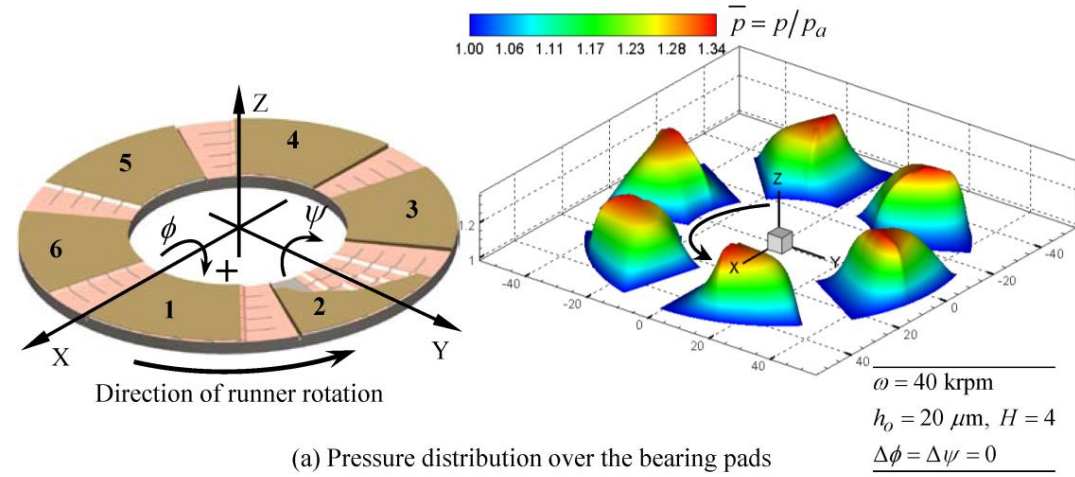



(b) Compliant structure deflection

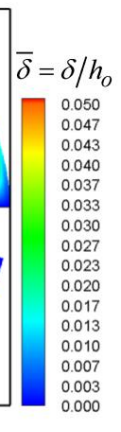

(c) Temperature distribution over the bearing pads

Fig. 3. Pressure distribution, bearing deformation, and temperature distribution of aligned foil thrust bearing

The pressure distribution in the gas film, the deflection of the compliant structure, and the temperature distribution in the gas film over all bearing pads are shown in Figure 3; the predictions are for a runner rotation speed of $40 \mathrm{krpm}$, nominal clearance $\left(h_{O}\right)$ of $0.02 \mathrm{~mm}$, and with statically aligned runner $(\phi=\psi=0)$. As expected, the results are identical for all bearing pads. For the above mentioned operating conditions, the maximum gas film pressure is about 1.35 times the ambient pressure and is located near the outer region of the bearing as a result of the runner velocity gradient in the radial direction. Also, the maximum temperature is about $32{ }^{\circ} \mathrm{C}$, and the maximum structural deformation is about 0.05 times the nominal clearance. Furthermore, the temperature distribution in the gas film, away from the converging region, varies in radial direction only since the variation of the gas film thickness in the circumferential direction is negligibly small. Moreover, the results show that the deflection of the compliant structure is very small and its maximum is attained near the free ends of the bump foil strips.

Figure 4 shows the variations of the gas film dynamic coefficients with the bearing load carrying capacity without runner misalignment $(\phi=\psi=0)$. The results are for a runner rotation speed of $40 \mathrm{krpm}$, inlet gas temperature of $25{ }^{\circ} \mathrm{C}$, and a maximum nominal clearance $\left(h_{\max }\right)$ of $0.03 \mathrm{~mm}$. The variations of the axial force stiffness coefficient $(K z z)$, axial force damping coefficient ( $C z z$ ), and the viscous power loss in the gas film with the bearing load carrying capacity is shown in Figure 4(a). It is clear from the Figure 4(a) that both $K z z$ and $C z z$ of the gas film increases almost linearly as the nominal film thickness 
ratio $\left(h_{o} / h_{\max }\right)$ decreases and the bearing load increases. In other words, for a 17 percent decrease of the gas film thickness, the value of $K z z$ and $C z z$ increase by 75 percent and 125 percent, respectively, at moderate to large bearing loads. This is attributed mainly to the enhanced hydrodynamic effects of foil thrust bearings with very thin gas films $[4,10$, 13]; as the nominal clearance decreases, the gas film becomes relatively stiff that it prevents further energy dissipation in the gas film.



(a) variation of axial force stiffness and damping coefficients with the bearing load

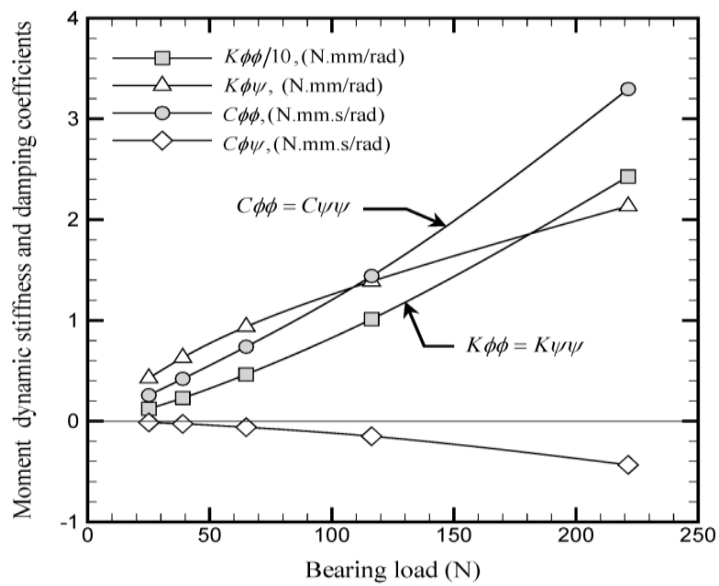

(b) variation of moment stiffness and damping coefficients with the bearing load

Fig. 4. Variation of the static and dynamic characteristics with bearing load for aligned foil thrust bearing

Figure 4(b) shows the variations of the gas film moment coefficients with bearing load carrying capacity. It is noticeable from the figure that the behavior of the direct moment stiffness and damping coefficients ( $K \phi \phi=K \psi \psi$ and $C \phi \phi=C \psi \psi$ ) when increasing the bearing load carrying capacity (i.e., decreasing the nominal clearance) resembles that of $K z z$ and $C z z$. On the other hand the cross-coupled moment stiffness coefficients 
( $K \phi \psi=-K \psi \phi)$ and the cross-coupled moment damping coefficients $(C \phi \psi=-C \psi \phi)$ increases in a relatively slower way with increasing the bearing load. This behavior of the cross-coupled dynamic coefficients demonstrates the stability of foil thrust bearings when operating at very thin gas films (i.e., higher bearing loads) since the cross-coupled coefficients are responsible for the non-restorative as well as non-dissipative forces in the gas film. As the values of the dynamic coefficients $K z \phi, K \phi z, C z \phi$ and $C \phi z$ are negligibly small for statically aligned foil thrust bearing [10, 12], their variation is not shown here.

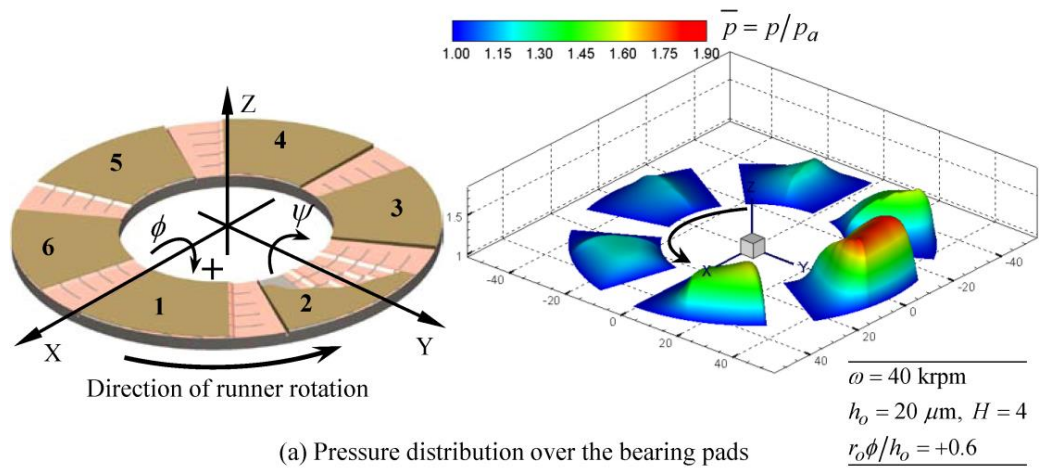

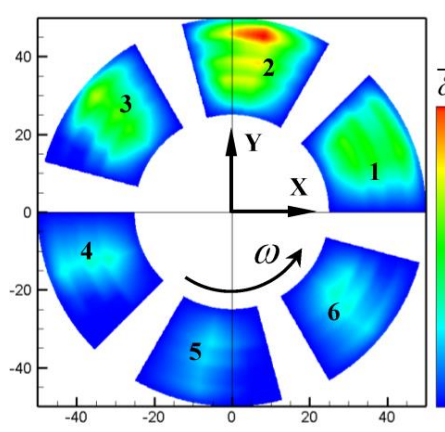

(b) Compliant structure deflection
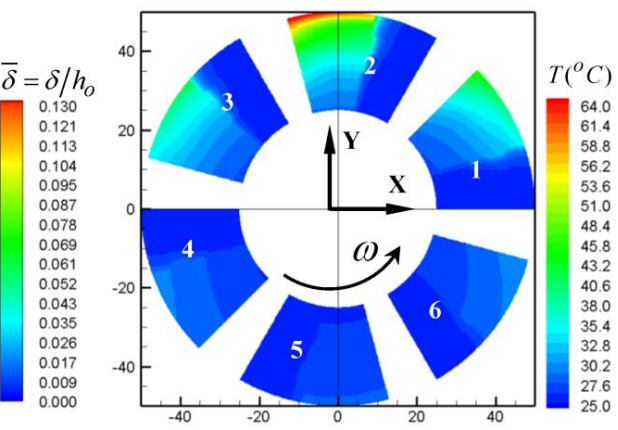

(c) Temperature distribution over the bearing pads

Fig. 5. Pressure distribution, bearing deformation, and temperature distribution of misaligned foil thrust bearing

\subsection{Static characteristics with misaligned shaft collar}

For the results presented in this section, the runner surface is allowed to tilt around the $\mathrm{X}$-axis only in the positive direction shown in Figure 5(a). Figure 5 shows the pressure distribution in the gas film, the deflection of the compliant structure, and the temperature distribution in the gas film over all bearing pads. These predictions are for a runner rotation speed of $40 \mathrm{krpm}$, nominal clearance $\left(h_{o}\right)$ of $0.02 \mathrm{~mm}$, and a static angular misalignment $(\Delta \phi)$ of 240 micro-radians in a clockwise direction which corresponds to a minimum gas film thickness as low as 40 percent of the nominal clearance; the inlet gas temperature is set at $25^{\circ} \mathrm{C}$. As a consequence of such static angular misalignment about the $\mathrm{X}$-axis, the thickness of the gas film in the gap between the rotating runner and the top foil varies in both radial and circumferential directions. Additionally, the gas film thickness over bearing pads No.1, 2, and 3 are smaller than the initial nominal clearance and the bearing pad No. 2 experiences the minimum gas film thickness (see Figure 5(a) for pad 
index numbers), and hence a larger gas film pressure. On the other hand, the bearing pads at the opposite side of the thrust plate (i.e., pads No. 4, 5, and 6) exhibits considerably larger gas film thicknesses and, consequently, smaller hydrodynamic pressure.

The following performance features can be noticed from the predictions shown in Figure 5: (1) the peak pressure in the gas film of bearing pads No.1,2, and 3 is displaced towards the outer rim of the thrust plate because of the radially-decreasing gas film thickness, (2) as the gas film temperature is inversely proportional to the gas film thickness, the maximum film temperature is located near the outer rim of bearing pad No.2 and its value, $64^{\circ} \mathrm{C}$, is about 2 times that with aligned runner surface, (3) the maximum deflection of the compliant structure is less than 15 percent of the nominal clearance and is about 3 times that with aligned runner surface, (4) the bearing pads No.1, 2, and 3 support most of the bearing load and their bearing surface deflects to accommodate the mechanical distortion while maintaining a sufficient hydrodynamic effect.

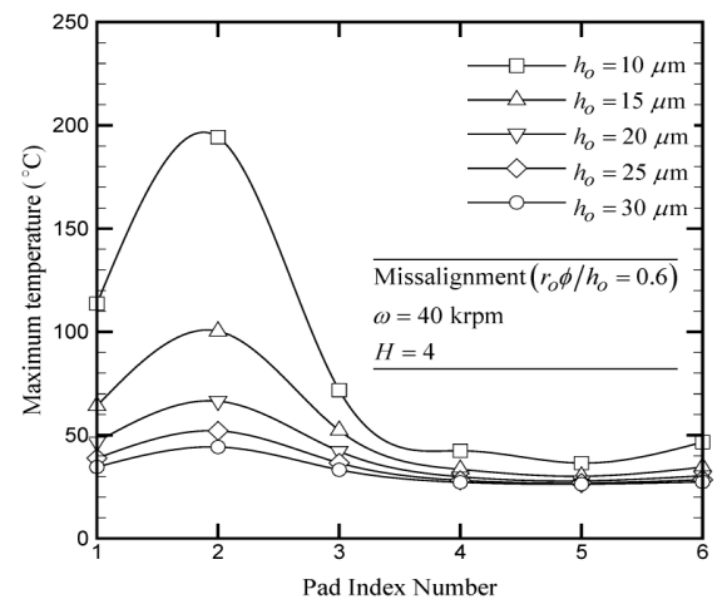

Fig. 6. Variation of maximum film temperature with nominal clearance over all bearing pads

Figure 6 shows the predicted maximum gas film temperature over all bearing pads for different nominal clearances $\left(h_{o}\right)$ and with a static angular misalignment factor of 0.6 that yields a minimum gas film thickness as low as 40 percent of the nominal clearance; the rotation speed is $40 \mathrm{krpm}$ and the inlet gas temperature is $25^{\circ} \mathrm{C}$. The figure shows that the gas film of the bearing pad No.2 experiences the maximum temperature while that of bearing pad No.5 experiences the minimum temperature. Further, maximum gas film temperature increases as the nominal clearance decreases. These results are mainly attributed to the higher energy dissipation in the gas film as its thickness is decreased.

The variations of the bearing load carrying capacity, power loss, and restoring moments ( $M X$ and $M Y$ ) with the misalignment factor $\left(r_{o} \phi / h_{o}\right)$ at a rotation speed of $40 \mathrm{krpm}$ and with two different nominal clearances of $0.01 \mathrm{~mm}$ and $0.03 \mathrm{~mm}$ is shown in Figure 7(a) and Figure 7(b), respectively. 


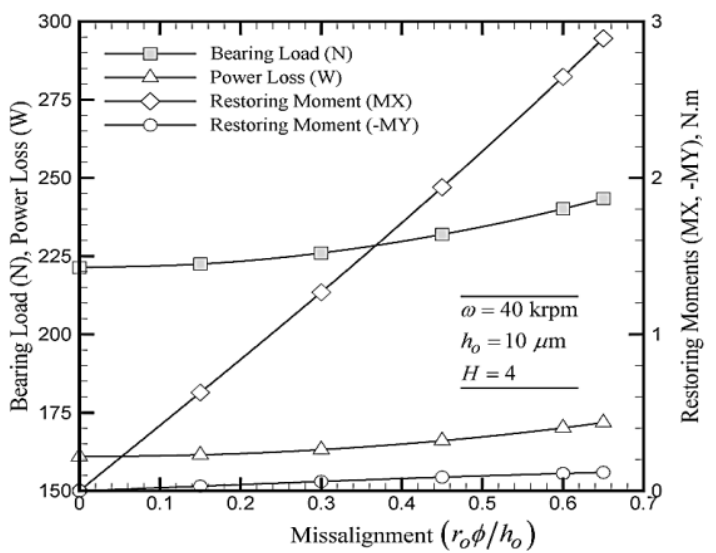

(a) variation of the bearing load, power loss, and restoring moments with misalignment factor, $\left(h_{O}=0.01 \mathrm{~mm}\right)$



(b) variation of the bearing load, power loss, and restoring moments with misalignment factor, $\left(h_{o}=0.03 \mathrm{~mm}\right)$

Fig. 7. Variation of bearing static characteristics with misalignment factor $\left(r_{O} \phi / h_{O}\right)$

It is clear from Figure 7 that both the load carrying capacity and the power dissipation in the gas film increase slowly with increasing the static misalignment of the runner; this is attributed to the fact that the increase of the load carrying capacity and power dissipation at one side of the bearing is accompanied by a corresponding decrease at the other side. However, the behavior of the main restoring moment ( $M X)$ of very thin gas films differs significantly from that of relatively thick gas films. For operation with thin gas film ( $h_{o}=0.01 \mathrm{~mm}$ ), the restoring moment $(M X)$ increases linearly with increasing the misalignment factor while it has a sluggish increase with thick gas film ( $\left.h_{O}=0.03 \mathrm{~mm}\right)$; the value of the restoring moment of the thin gas film is about 4 times that of the thick gas film at a misalignment factor of 0.6. This behavior demonstrates the ability of the thin gas film to withstand such imposed mechanical distortions much more than the thick gas films. The restoring moment ( $M Y$ ) shows a very slow increase when increasing the misalignment factor and the reason behind this behavior is the asymmetrical distribution of the bearing pads about the $\mathrm{Y}$-axis, see Figure 5(a). 


\subsection{Dynamic characteristics with misaligned shaft collar}

The results presented in this section were obtained at a rotation speed of $40 \mathrm{krpm}$ and nominal clearance $\left(h_{o}\right)$ of $0.02 \mathrm{~mm}$ while applying a small dynamic misalignments ( $\Delta \phi, \Delta \psi$, and $\Delta z$ ). The variations of the force stiffness and force damping coefficients with the static misalignment factor are shown in Figure 8(a) and Figure 8(b), respectively. These results can be interpreted by calling the definition of the various dynamic coefficients as follows:

$$
\begin{aligned}
& K i j=-\partial F i / \partial \Delta j, \\
& C i j=-\partial F i / \partial \dot{\Delta} j,
\end{aligned} \quad i, j=\phi, \psi, z
$$

Hence, the force stiffness coefficient $K z \phi$ corresponds to a stiffness produced by a gas film force in Z-direction as a result of runner misalignment (rotation) around the $\mathrm{X}$-axis; here, the runner misalignment is composed of static and dynamic contributions.



(a) variation of force stiffness coefficients with misalignment factor



(b) variation of force damping coefficients with misalignment factor

Fig. 8. Variation of force stiffness and damping coefficients with misalignment factor $\left(r_{o} \phi / h_{O}\right)$ 
The results from Figure 8 show that, for a small perturbations ( $\Delta \phi, \Delta \psi$, and $\Delta z$ ) about the equilibrium position of a statically-misaligned runner, the direct stiffness and direct damping coefficients ( $K z z$ and $C z z$ ) increase nonlinearly with the increase of the static misalignment factor. Furthermore, the results show clearly that the combined effects of the static and dynamic misalignments about the $\mathrm{X}$-axis resulted in non-restorative as well as non-dissipative forces in the gas film which are manifested by the rapid increase of the cross-coupled stiffness and damping coefficients ( $K z \phi$ and $C z \phi$ ); however, their values still very small when compared with those of the direct coefficients ( $K z z$ and $C z z$ ) and, consequently, the rotordynmaic stability is maintained. Also, it can be noticed from Figure 8(a) that the force stiffness coefficient $(K z \psi)$ is less sensitive to the static misalignment factor $\left(r_{o} \phi / h_{o}\right)$; this is mainly attributed to the fact that there is no static misalignment around the $\mathrm{Y}$-axis $(\psi=0)$ and hence, the shown increase in $K z \psi$ is due to the increase of the bearing pressure that results from the static misalignment around the $\mathrm{X}$ axis. In a similar way, the increase of the force damping coefficient ( $C z \psi$ ) with misalignment factor can be interpreted.

The variations of the moment stiffness and moment damping coefficients with the static misalignment factor are shown in Figure 9(a) and Figure 9(b), respectively. It is apparent from Figure 9 that direct stiffness coefficients ( $K \phi \phi$ and $K \psi \psi$ ) are no longer equal, and so does the direct damping coefficients ( $C \phi \phi$ and $C \psi \psi$ ), at moderate to high misalignment factors; further, the values of the coefficient ( $K \phi \phi$ and $C \phi \phi$ ) are considerably higher than those of the coefficients ( $K \psi \psi$ and $C \psi \psi$ ) at this region. The reason behind this deviation is the contribution of the static misalignment about $\mathrm{X}$-axis that strongly affects the coefficients ( $K \phi \phi$ and $C \phi \phi)$. Another important notice from Figure 9(a) is that the crosscoupled stiffness coefficients ( $K \phi \psi$ and $K \psi \phi$ ) are skew symmetric and equal (i.e., $K \phi \psi=-K \psi \phi$ ). Similarly, Figure 9(b) shows that the cross-coupled damping coefficients ( $C \phi \psi$ and $C \psi \phi$ ) are skew symmetric and equal (i.e., $C \phi \psi=-C \psi \phi$ ). As a result of the efficient hydrodynamic effect, the values of the abovementioned cross-coupled coefficients are very small and less sensitive to the static misalignment factor.



(a) variation of moment stiffness coefficients with misalignment factor 


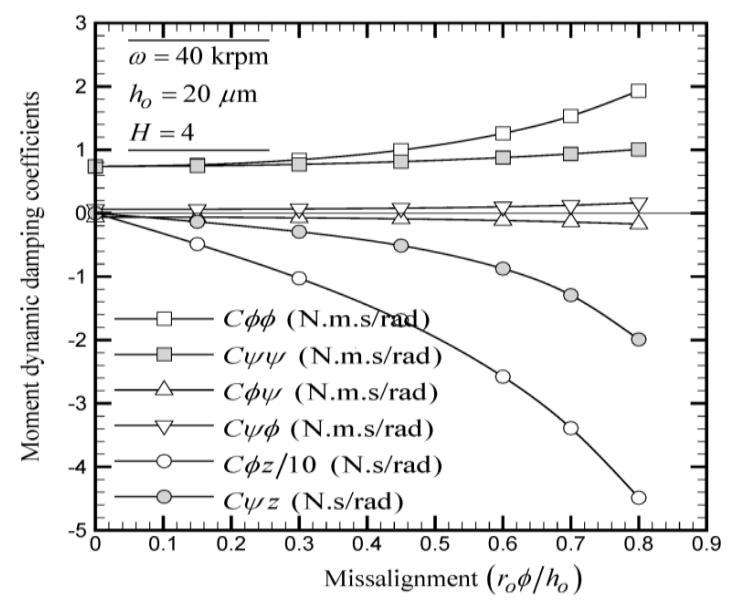

(b) variation of moment damping coefficients with misalignment factor

Fig. 9. Variation of moment stiffness and damping coefficients with misalignment factor $\left(r_{O} \phi / h_{O}\right)$

Also, the data from Figure 9 shows that both the stiffness coefficient ( $K \phi z$ ) and the damping coefficient ( $C \phi z$ ) increase rapidly as the static misalignment factor increases however their negative effect on the bearing stability still very small compared with the positive effect of the direct stiffness and damping coefficients; a maximum static misalignment $(\phi)$ of 320 micro-radians would yield a net non-restorative force of less than $1.0 \mathrm{~N}$. These results show clearly that the foil thrust bearing can operate stably under conditions of static and dynamic misalignments about $\mathrm{X}, \mathrm{Y}$, and $\mathrm{Z}$ axes.

\section{Conclusions}

In the present numerical simulations, the effect of static and dynamic shaft collar (runner) misalignment on the performance of gas-lubricated bump-type foil thrust bearing was analyzed. The two-dimensional compressible Reynolds equation was used to model the flow in the gas film between the runner and top foil surfaces and the Couette approximation technique is used to calculate the adiabatic temperature distribution in the gas film. The dynamic force and moment coefficients were calculated using the small perturbations method. The performance characteristics of aligned as well as misaligned foil thrust bearing were evaluated. The following concluding remarks could be obtained from the present predictions:

- The peak pressure and the maximum temperature in the gas film are located near the outer rim of the bearing pad that experiences the largest misalignment effect.

- The load carrying capacity of the bearing and the power dissipation in the gas film show a sluggish increase with increasing the static misalignment of the runner. Also, the principal restoring moment of the thin gas films is much higher than that of thick gas films

- The direct force and moment coefficients increase nonlinearly with increasing the misalignment of the runner. Furthermore, the effects of the coefficients that are 
responsible for non-restorative as well as non-dissipative forces in the gas film are negligibly small.

\section{REFERENCES}

[1] Heshmat H, Walowit JA and Pinkus O. Analysis of Gas-Lubricated Compliant Thrust Bearings. J Lubr Technol 1983; 105: 638-646.

[2] Heshmat H, Walowit JA and Pinkus O. Analysis of Gas-Lubricated Foil Journal Bearings. J Lubr Technol 1983; 105: 647-655.

[3] Agrawal G L. Foil Air/Gas Bearing Technology - An Overview. International Gas Turbine \& Aeroengine Congress \& Exhibition, 1997, ASME Paper No. 97-GT-347.

[4] Dellacorte C and Valco MJ. Load Capacity Estimation of Foil Air Journal Bearings for OilFree Turbomachinery Applications. Tribol Trans 2000; 43 (4): 795-801.

[5] Gu A and Nadjafi R, 1993. Foil Thrust Bearing With Varying Radial and Circumferential Stiffness. U.S. Patent No. 5,248,205.

[6] Heshmat H, 1997. High load capacity compliant foil hydrodynamic thrust bearing. U.S. Patent No. 5,833,369.

[7] Heshmat CA, Xu DS and Heshmat H. Analysis of Gas Lubricated Foil Thrust Bearings Using Coupled Finite Element and Finite Difference Methods. J Tribol 2000; 122 (1): 199-204.

[8] Iordanoff I. Analysis of an Aerodynamic Compliant Foil Thrust Bearing: Method for a Rapid Design. J Tribol 1999; 121 (4): 816-822.

[9] Bruckner RJ. Simulation and Modeling of the Hydrodynamic, Thermal, and Structural Behavior of Foil Thrust Bearings. PhD Thesis, Case Western Reserve University, USA, 2004.

[10] Lee DH and Kim DJ. Design and Performance Prediction of Hybrid Air Foil Thrust Bearings. J Eng Gas Turbines Power, 2011; 133: 042501.

[11] Lee DH and Kim DJ. Three-Dimensional Thermohydrodynamic Analyses of Rayleigh Step Air Foil Thrust Bearing with Radially Arranged Bump Foils. Tribol Trans, 2011; 54: 432-448.

[12] Gad AM and Kaneko S. A New Structural Stiffness Model for Bump-type Foil Bearings: Application to Generation II Gas Lubricated Foil Thrust Bearing. J Tribol 2014; 136 (4): 041701.

[13] Gad AM and Kaneko S. Performance characteristics of gas-lubricated bump-type foil thrust bearing. Proc IMechE Part J: Journal of Engineering Tribology, 2014; 229(6): 746762. DOI: $10.1177 / 1350650114564709$.

[14] Gad AM and Kaneko S. Fluid Flow and Thermal Features of Gas Foil Thrust Bearings at Moderate Operating Temperatures," Proceedings of the 9th IFToMM International Conference on Rotor Dynamics, Mechanisms and Machine Science, 2015; Vol. 21: 12231233, DOI. 10.1007/978-3-319-06590-8_100

[15] Gad AM and Kaneko S. Tailoring of the Tailoring of the Bearing Stiffness to Enhance the Performance of Gas-Lubricated Bump-Type Foil Thrust Bearing. Proc IMechE Part J: Journal of Engineering Tribology, Pub a head of print, 2015; DOI: $10.1177 / 1350650115606482$.

[16] Dykas BD. Factors Influencing the Performance of Foil Gas Thrust Bearings for Oil-Free Turbomachinery Applications. PhD Thesis, Case Western Reserve University, USA, 2006.

[17] Dickman JR. An Investigation of Gas Foil Thrust Bearing Performance and Its Influencing Factors. MSc Thesis, Case Western Reserve University, USA, 2010.

[18] Bruckner R J. Performance of Simple Gas Foil Thrust Bearing in Air. tech. report, NASA/TM - 2012-217262.

[19] Park DJ, Kim CH, Jang GH, and Lee YB. Theoretical Considerations of Static and Dynamic Characteristics of Air Foil Thrust Bearing with Tilt and Slip Flow. Tribol Int 2008; 41: 282-295.

[20] Pinkus O and Bupara SS. Adiabatic Solutions for Finite Journal Bearings. J Lubr Technol 1979; 101: 492-496.

[21] Salehi M, Swanson E, and Heshmat H. Thermal Features of Compliant Foil Bearings Theory and Experiments. J Tribol 2001; 123: 566-571. 
[22] Walowit J A and Anno J N. Modern Developments in Lubrication Mechanics. London: Applied Science Publishers Ltd; 1975.

[23] Ku CR and Heshmat H. Compliant Foil Bearing Structural Stiffness Analysis: Part I- Theoretical Model Including Strip and Variable Bump Foil Geometry. J Tribol 1992; 114: 394-400.

[24] Le Lez S, Arghir M and Frene J. A New Bump-Type Bearing Structure Analytical Model. J Eng Gas Turbines Power 2007; 129 (4): 1047-1057.

[25] Feng K and Kaneko S. Analytical Model of Bump-Type Foil Bearings Using a LinkSpring Structure and a Finite-Element Shell Model. J Tribol 2010; 132 (2): 021706.

[26] Hryniewicz P, Wodtke M, Olszewski A, and Rzadkowaski R. Structural Properties of Foil Bearings: A Closed-Form Solution Validated with Finite Element Analysis. Tribol Trans 2009; 52: 435-446.

[27] Kim D, Ezeka C and Kumar M. Revisit to Stiffness of Bump Foils in Air Foil Bearings. ASME/STLE International Joint Tribology Conference, 2007, paper no. IJTC2007-44319.

\section{Nomenclature}

$C$ : damping coefficient

$C_{p}:$ gas specific heat, $\mathrm{J} / \mathrm{kg} \cdot{ }^{o} \mathrm{~K}$

$E$ : Young's modulus of bump foil, GPa

$F$ : load carrying capacity, $\mathrm{N}$

$G$ : non dimensional auxiliary function

$h$ : film thickness, $\mathrm{m}$

$\bar{h}$ : dimensionless film thickness, $\bar{h}=h / h_{O}$

$h_{1}$ : ramp height, $\mathrm{m}$

$h_{o}$ : nominal film thickness, $\mathrm{m}$

$h_{b}$ : bump height, $\mathrm{m}$

$H$ : film thickness ratio, $H=h_{1} / h_{o}$

$K$ : stiffness coefficient

$L_{b}$ : bump length, $\mathrm{m}$

$N$ : number of bumps

$p$ : gas film pressure, $\mathrm{N} / \mathrm{m}^{2}$

$P w$ : power dissipation in the gas film, $\mathrm{W}$

$p_{a}:$ ambient pressure

$\bar{p}$ : dimensionless pressure, $\bar{p}=p / p_{a}$

$p_{z}, p_{\dot{z}}, p_{\phi}, p_{\dot{\phi}}, p_{\psi}, p_{\dot{\psi}}$ : pressure perturbations.

$R$ : bump radius of curvature, $\mathrm{m}$

$r$ : radial coordinate, $\mathrm{m}$

$r_{i}$ : inner radius of the bearing, $\mathrm{m}$

$r_{O}$ : outer radius of the bearing, $\mathrm{m}$

$\bar{r}$ : dimensionless radial distance, $\bar{r}=r / r_{o}$
$T$ : gas film temperature, ${ }^{\circ} \mathrm{C}$

$t$ : time variable, sec.

$\bar{t}$ : normalized time variable, $\bar{t}=t v$

$t_{b}$ : bump foil thickness, $\mathrm{m}$

$z \quad$ : coordinate across the film thickness, $\mathrm{m}$

$\Delta z, \Delta \phi, \Delta \psi, \Delta \dot{z}, \Delta \dot{\phi}, \Delta \dot{\psi}:$ displacement and velocity perturbations

$\alpha, \beta$ : viscosity-temperature relation coefficients

$\delta$ : bump deflection, $\mathrm{m}$

$\bar{\delta}$ : dimensionless bump deflection, $\bar{\delta}=\delta / h_{O}$

$\mu$ : gas viscosity, N.s $/ \mathrm{m}^{2}$

$\mu_{a}:$ air viscosity, N.s $/ \mathrm{m}^{2}$

$\bar{\mu}$ : dimensionless viscosity, $\bar{\mu}=\mu / \mu_{a}$

$\rho_{a}:$ air density, $\mathrm{kg} / \mathrm{m}^{3}$

$v \quad:$ whirl speed, $\mathrm{rad} / \mathrm{sec}$.

$v$ : Poisson's ratio of bump foil

$\Lambda$ : bearing number

$\gamma:$ whirl frequency ratio, $\gamma=v / \omega$

$\theta$ : angular coordinate

$\phi \quad$ : static angular misalignment about $\mathrm{X}$-axis

$\psi \quad$ : static angular misalignment about $\mathrm{Y}$-axis

$\theta_{c}, \theta_{p}$ : angular extensions of converging region and bearing pad, respectively, degrees $\omega$ : rotation velocity, $\mathrm{rad} / \mathrm{sec}$. 
تأثير المحاذاة الخاطئة على ثبات كرسي تحميل دفعي ذو رقائق معدنية مزيت بالغاز

$$
\text { ملخص البحث: }
$$

تهدف الدراسة الحالية إلى تقييم تأثثر الإنحر اف الزاوي الإستاتيكي والديناميكي للسطح الدوار على دإنى أداء



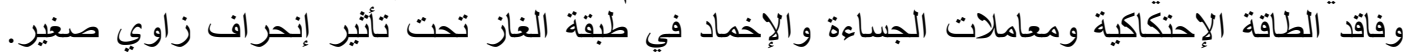

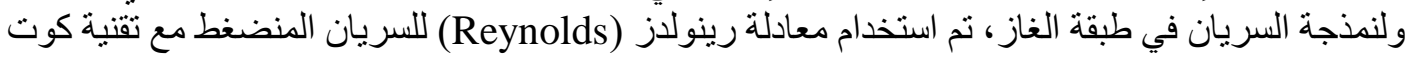

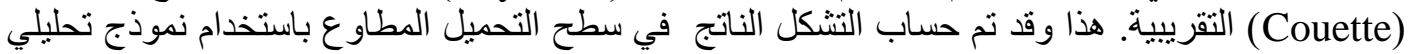



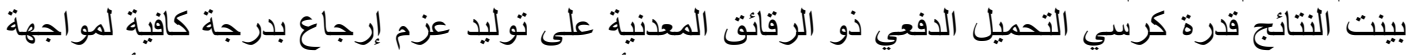

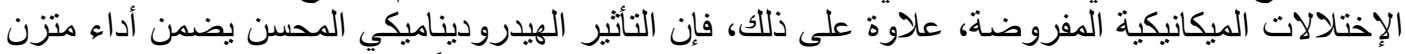

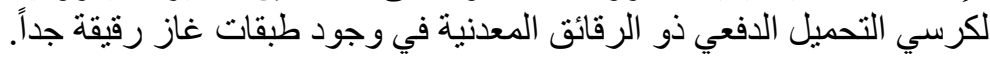

